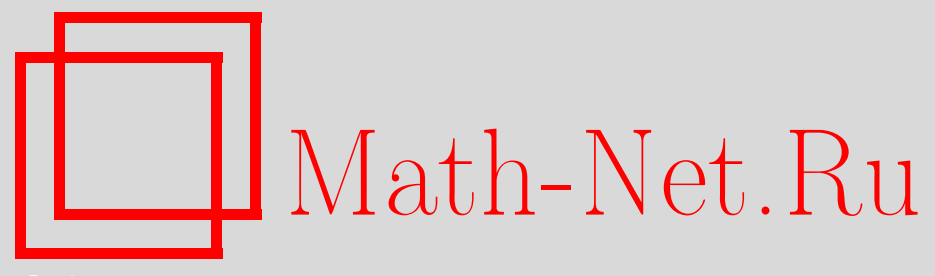

Е. В. Мурашкин, Ю. Н. Радаев, Об определяющих псевдоскалярах гемитропных микрополярных сред в инверсных координатных системах, Bестн. Сам. гос. техн. ун-та. Сер. Физ.-мат. науки, 2021, номер 3, 457-474

DOI: https://doi.org/10.14498/vsgtu1870

Использование Общероссийского математического портала MathNet.Ru подразумевает, что вы прочитали и согласны с пользовательским соглашением

http: //www . mathnet.ru/rus/agreement

Параметры загрузки:

IP : 52.23 .180 .231

26 апреля 2023 г., 07:37:45

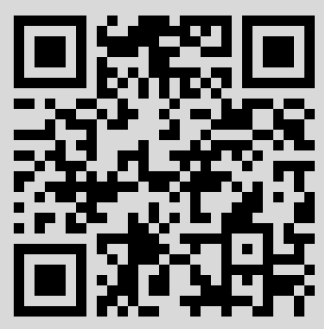


УДК 539.3

\title{
Об определяющих псевдоскалярах гемитропных микрополярных сред в инверсных координатных системах
}

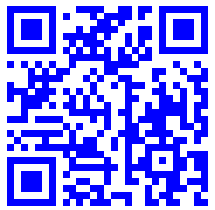

\section{(C) Е. В. Мурашкин, Ю. Н. Радаев}

Институт проблем механики им. А. Ю. Ишлинского РАН, Россия, 119526, Москва, просп. Вернадского, 101, корп. 1.

\begin{abstract}
Аннотация
Обсуждаются определяющие псевдоскаляры, связанные с теорией гемитропного микрополярного континуума. Приводятся основные понятия алгебры псевдотензоров. Определяется псевдотензорная форма гемитропного микрополярного упругого потенциала, основанная на 9 определяющих псевдоскалярах (из них 3 псевдоскаляра и 6 абсолютных скаляров). Вычисляются веса определяющих псевдоскаляров. С помощью фундаментального ориентирующего псевдоскаляра веса +1 формулируются правила преобразования определяющих псевдоскаляров. Выводятся определяющие уравнения гемитропного микрополярного упругого континуума. Обсуждаются уравнения динамики гемитропного микрополярного континуума в терминах псевдотензоров в право- и левоориентированных декартовых системах координат. Показано наличие инверсных мод наряду с прямыми при распространении волн по гемитропному микрополярному континууму.
\end{abstract}

Ключевые слова: микрополярный гемитропный континуум, микроповорот, псевдоскаляр, относительный тензор, фундаментальный ориентирующий псевдоскаляр, определяющее уравнение, инверсия пространства, поляризация.

Получение: 23 июня 2021 г. / Исправление: 29 июля 2021 г. Принятие: 25 августа 2021 г. / Публикация онлайн: 27 сентября 2021 г.

\footnotetext{
Научная статья

( () (7) Контент публикуется на условиях лицензии Creative Commons Attribution 4.0 International (https://creativecommons.org/licenses/by/4.0/deed.ru)

Образец для цитирования

М у раш к и н Е. В., Р а да ев Ю. Н. Об определяющих псевдоскалярах гемитропных микрополярных сред в инверсных координатных системах // Вестн. Сам. гос. техн. ун-та. Сер. Физ.-мат. науки, 2021. Т. 25, № 3. С. 457-474. https://doi.org/10.14498/vsgtu1870.
}

\section{Сведения об авторах}

Евгений Валеръевич Мурашкин (10) https://orcid.org/0000-0002-3267-4742

кандидат физико-математических наук; старший научный сотрудник; лаб. моделирования в механике деформируемого твердого тела; e-mail: evmurashkin@google.com

Юрий Николаевич Радаев (1) https://orcid.org/0000-0002-0866-2151

доктор физико-математических наук, профессор; ведущий научный сотрудник; лаб. моделирования в механике деформируемого твердого тела; e-mail:radayev@ipmnet.ru, y.radayev@gmail.com 
Введение. Современные конструкционные и метаматериалы обладают такими физико-механическими свойствами, о существовании которых нельзя было даже подозревать еще несколько десятилетий назад: отрицательный коэффициент Пуассона (ауксетические материалы), отрицательное тепловое расширение, отрицательная электрическая и магнитная проницаемость. Метаматериал демонстрирует характеристики отклика, которые либо не наблюдаются, либо усиливаются по сравнению с индивидуальными откликами составляющих его материалов. Сотовые конструкции являются системами из хорошо известных конструкционных элементов, в целом могут проявлять нестандартное поведение в ответ на механические воздействия. Биологические ткани животного происхождения (мышечная ткань, длинные (трубчатые) кости, стенки кровеносных сосудов) проявляют ярко выраженные гемитропные свойства, что подтверждается многочисленными исследованиями [1-3]. Поэтому при математическом моделировании процессов деформирования и роста таких материалов необходимо понимать, что классические модели механики сплошных сред будут накладывать чрезмерные ограничения. При построении таких моделей важно соблюдать геометрическую и термодинамическую непротиворечивость. Механические свойства материалов, проявляющих гемитропные свойства, зависят от зеркальных отражений микроструктурного состояния микрополярного упругого тела. Последовательное применение принципа виртуальных перемещений и алгебры псевдотензоров [4-10] в механике микрополярного континуума приводит к физически и геометрически корректным формулировкам определяющих уравнений.

Отметим, что построение определяющего упругого потенциала для гемитропного континуума возможно исключительно при использовании псевдотензорных формулировок, только после этого возможен корректный переход к абсолютным тензорам и вывод всех основных уравнений механики гемитропных тел. Еще одним существенным аспектом оперирования с уравнениями гемитропного тела является необходимость постоянно согласовывать баланс весов, особенно при использовании символов перестановок, которые можно трактовать одновременно как псевдотензоры весов +1 и -1 . Для этих же символов (как хорошо известно [9]) нарушается стандартное правило жонглирования индексами.

Многочисленные руководства по тензорному исчислению чаще всего обходят стороной вопросы, связанные с алгеброй псевдотензоров [11]. Ранее в работах авторов [12-14] обсуждались вопросы применения алгебры псевдотензоров к задачам механики растущих тел и микрополярной теории упругости. В настоящей работе обсуждаются вопросы задания определяющих псевдоскаляров теории гемитропного континуума. Приводятся правила преобразования определяющих псевдоскаляров при изменении системы координат. Выводятся определяющие уравнения гемитропного микрополярного упругого континуума. Обсуждаются уравнения динамики гемитропного микрополярного континуума в терминах псевдотензоров для прямых и зеркальных мод. С целью компактности изложения используемые термины и обозначения сведены в табл. 1-3.

1. Основные сведения из алгебры псевдотензоров. Рассмотрим в $n$ мерном пространстве две системы координат $x^{k}$ и $\bar{x}^{k}(k=1,2, \ldots n)$. Преобразование относительного тензора веса $W$ (псевдотензора веса $W$ ) от системы 
координат $x^{k}$ к новой системе координат $\bar{x}^{k}$ осуществляется по закону $[15,16]$

$$
\bar{T}_{i j \cdots k}^{l m \cdots n}=\Delta^{W}\left(\partial_{p} \bar{x}^{l}\right)\left(\partial_{q} \bar{x}^{m}\right) \cdots\left(\partial_{s} \bar{x}^{n}\right)\left(\overline{\partial_{i}} x^{a}\right)\left(\overline{\partial_{j}} x^{b}\right) \cdots\left(\overline{\partial_{k}} x^{c}\right) T_{a b \cdots c}^{p q \cdots s}
$$

где

$$
\Delta=\operatorname{det}\left(\overline{\partial_{j}} x^{i}\right), \quad \partial_{p}=\frac{\partial}{\partial x^{p}}, \quad \overline{\partial_{p}}=\frac{\partial}{\partial \bar{x}^{p}} .
$$

Здесь черта сверху указывает на значение величины в новой системе координат $\bar{x}^{k}(k=1,2, \ldots n), \Delta$ - якобиан преобразования, $W$ - вес псевдотензора. Отметим, что закон преобразования псевдотензоров отличается от закона преобразования абсолютных тензоров дополнительным множителем $\Delta^{W}$; $W$ - целое число, так как в противном случае значение $\Delta^{W}$ не будет однозначным.

Для псевдотензоров справедливы следующие утверждения:

1) сумма двух псевдотензоров одинаковой валентности и веса будет псевдотензором той же валентности и веса:

$$
\stackrel{[W]}{A} \underset{k l}{i j}=\stackrel{[W]}{B} \underset{k l}{i j}+\stackrel{[W]}{C} \underset{k l}{i j}
$$

2) тензорное произведение псевдотензоров (возможно, различных валентностей) дает псевдотензор с итоговым весом, равным сумме весов сомножителей

$$
\stackrel{\left[W_{1}+W_{2}\right]_{i j p q s}}{A} \underset{\text { klrtm }}{B}=\stackrel{\left[W_{1}\right]}{B} \underset{k l}{i j} \stackrel{[W}{C}_{r t m}^{p q s}
$$

3) результатом свертки псевдотензора будет псевдотензор того же веса; в том числе, полная свертка псевдотензора веса $W$ будет псевдоскаляром того же веса.

Одним из фундаментальных объектов многомерной геометрии является абсолютный тензор $\delta_{i_{1} i_{2} \ldots i_{k}}^{j_{1} j_{2} \ldots j_{k}}$, называемый обобщенной дельтой Кронекера и определяемый в $n$-мерном пространстве для $k \leqslant n$ по правилу

$\delta_{i_{1} i_{2} \ldots i_{k}}^{j_{1} j_{2} \ldots j_{k}}=\left\{\begin{aligned}+1, & \text { если } j_{1}, j_{2}, \ldots, j_{k} \text { - различные натуральные числа } 1,2, \ldots, n \\ & \text { и если } i_{1}, i_{2}, \ldots, i_{k} \text { - четная перестановка } j_{1}, j_{2}, \ldots, j_{k} ; \\ -1, & \text { если } j_{1}, j_{2}, \ldots, j_{k} \text { - различные натуральные числа } 1,2, \ldots, n \\ & \text { и если } i_{1}, i_{2}, \ldots, i_{k} \text { - нечетная перестановка } j_{1}, j_{2}, \ldots, j_{k} ; \\ 0, & \text { во всех остальных случаях. }\end{aligned}\right.$

С помощью тензора $\delta_{i_{1} i_{2} \ldots i_{k}}^{j_{1} j_{2} \ldots j_{k}}$ легко вычисляются символы перестановок:

1) относительный ковариантный $n$-вектор $\epsilon_{i_{1} i_{2} \ldots i_{n}}$ веса -1 :

$$
\epsilon_{i_{1} i_{2} \ldots i_{n}}=\delta_{i_{1} i_{2} \ldots i_{n}}^{12 \ldots n}
$$

2) относительный контравариантный $n$-вектор $\epsilon^{i_{1} i_{2} \ldots i_{n}}$ веса +1 :

$$
\epsilon^{i_{1} i_{2} \ldots i_{n}}=\delta_{12 \ldots n}^{i_{1} i_{2} \ldots i_{n}}
$$

Определение косого произведения $n$ абсолютных ковариантных векторов $\underset{1}{\mathbf{a}}, \underset{2}{\mathbf{a}}, \ldots, \underset{n}{\mathbf{a}}$ в $n$-мерном пространстве имеет вид [17]

$$
\lceil\underset{1}{\mathbf{a}}, \underset{2}{\mathbf{a}}, \ldots, \underset{n}{\mathbf{a}}\rfloor=e_{i_{1} i_{2} \cdots i_{n}} \underset{1}{a^{i_{1}}}{\underset{2}{i_{2}} \cdots}_{n}^{a_{n}^{i_{n}}}
$$


где $e_{i_{1} i_{2} \cdots i_{n}}=e \epsilon_{i_{1} i_{2} \cdots i_{n}}, e=e_{12 \cdots n}-$ псевдоскаляр веса +1 , позволяет ввести понятие фундаментального ориентирующего псевдоскаляра и разделить правые и левые локальные базисные системы. В самом деле, если в качестве системы векторов $\underset{1}{\mathbf{a}}, \underset{2}{\mathbf{a}}, \ldots, \underset{n}{\mathbf{a}}$ принять векторы ковариантного базиса $\underset{1}{\boldsymbol{\imath}}, \underset{2}{\boldsymbol{\imath}}, \ldots, \underset{n}{\boldsymbol{\imath}}$ в $n$-мерном пространстве, то на основании (2) находим

$$
\lceil\underset{1}{\boldsymbol{\imath}, \boldsymbol{\imath}}, \ldots, \underset{n}{\boldsymbol{\imath}}\rfloor=e \operatorname{det}\left(\underset{a}{\imath^{c}}\right)
$$

Заметим, что вектор $\underset{a}{\boldsymbol{\imath}}$ может быть разложен по базису $\underset{c}{\boldsymbol{\imath}}$

$$
\underset{a}{\boldsymbol{\imath}}=\underset{a}{\imath^{c}} \boldsymbol{c},
$$

откуда

$$
\underset{a}{\imath^{c}}=\underset{a}{\underset{\imath}{\boldsymbol{\imath}}} \stackrel{c}{=} \underset{\mathrm{\delta}}{\mathrm{c}}
$$

откуда

$$
\operatorname{det}\left(\imath_{a}^{c}\right)=\operatorname{det}(\stackrel{c}{\delta})=1
$$

т.e.

$$
\lceil\underset{1}{\boldsymbol{\imath}}, \underset{2}{\boldsymbol{\imath}}, \ldots, \underset{n}{\boldsymbol{\imath}}\rfloor=e
$$

В трехмерном пространстве $e$ определяется смешанным произведением базисных векторов:

$$
e=\stackrel{[+1]}{e}=\lceil\underset{1}{\boldsymbol{\imath}}, \underset{2}{\boldsymbol{\imath}}, \underset{3}{\boldsymbol{\imath}}\rfloor=\underset{1}{\boldsymbol{\imath}} \cdot(\underset{2}{\boldsymbol{\imath}} \times \underset{3}{\boldsymbol{\imath}}),
$$

а фундаментальный ориентирующий псевдоскаляр отрицательного веса -1 есть

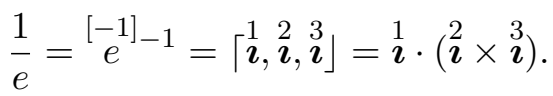

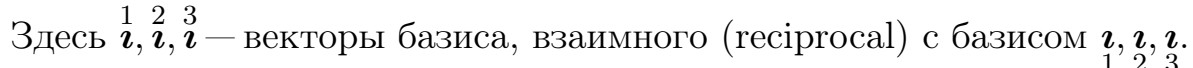

Отметим, что фундаментальный ориентирующий псевдоскаляр позволяет легко преобразовывать псевдотензоры в абсолютные тензоры. Введем тензор $\mathbf{T}$ согласно

$$
\mathbf{T}=e^{-W^{[W]}} \mathbf{T}
$$

Подсчитывая баланс весов, приходим к заключению, что Т является абсолютным тензором.

Известное в тензорной алгебре уравнение Гамильтона-Кэли остается справедливым для псевдотензоров второго ранга произвольного веса. Для доказательства этого утверждения введем комитанты $\underset{k}{C} \underset{k}{i} \cdot j$ и инвариантны $\underset{k}{\mathrm{I}}$ псевдоаффинора $\stackrel{[W]}{T} \underset{. j}{i}$, согласно формулам [5]

$$
k ! \stackrel{[k W]}{C} \underset{k}{i \cdot j}=(-1)^{k}(k+1) \delta_{j_{1} j_{2} \ldots j_{k} j}^{i_{1} i_{2} \ldots i_{k} i} \stackrel{[W]}{T}_{i_{1}}^{j_{1}} \cdot \stackrel{[W]}{T}{ }_{\cdot i_{2}}^{j_{2}} \cdots \stackrel{[W]}{T}_{j_{k}}^{j_{k}}
$$




$$
k ! \stackrel{[k W]}{\mathrm{I}}=\delta_{j_{1} j_{2} \ldots j_{k}}^{i_{1} i_{2} \ldots i_{k}}{\stackrel{[W]}{T_{i_{1}}}}_{j_{1}}^{\left[{ }^{[W]}\right.} \stackrel{[}{T}_{i_{2}}^{j_{2}} \ldots \stackrel{[W]}{T}_{\cdot i_{k}}^{j_{k}}
$$

Можно показать [18], что комитанты (4) выражаются через псевдоинварианты (5) и степени псевдоаффинора зависимостью

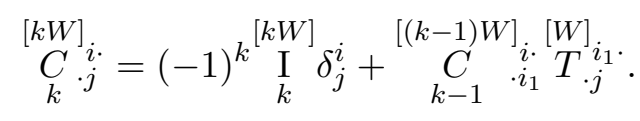

В итоге при $k=n$ правая часть равенства (4) содержит операцию альтернирования по $n+1$ индексу и поэтому равна нулю:

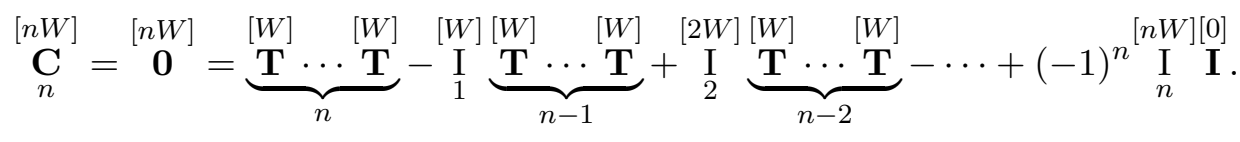

Соотношение (6) означает справедливость уравнения Гамильтона-Кэли для псевдотензоров в случае $n$-мерного пространства.

2. Определяющие уравнения гемитропного микрополярного тела. Псевдотензорная формулировка. Динамические уравнения гемитропного микрополярного тела в подавляющем большинстве литературных источников выводятся в терминах абсолютных тензоров [19-21]. Однако, как показали недавние исследования $[12,14]$, геометрически и физически корректная формулировка уравнений гемитропной микрополярной теории возможна только в терминах относительных тензоров.

Следствием принципа виртуальных перемещений $[14,22]$ являются уравнения динамики микрополярной среды, которые примем в форме

$$
\nabla_{i} t^{i k}=\rho \partial_{. .}^{2} u^{k}, \quad \nabla_{i}{ }^{[-1]_{i \cdot}} \cdot 2^{[-1]} \tau_{k}=\rho \stackrel{[-2]}{\Im} \partial_{. .}^{2[+1]} \phi_{k} \text {, }
$$

где ${ }^{[-1]}{ }_{j}$ - ассоциированный (сопутствующий) вектор силовых напряжений

$$
-\stackrel{[-1]}{\tau}_{j}=\frac{1}{2} \epsilon_{j i k} t^{[i k]}, \quad t^{[i k]}=-\epsilon^{i k j}{ }^{[-1]}{ }_{j} .
$$

Ассоциированный (сопутствующий) вектор моментных напряжений определяется по аналогии с (7):

$$
\mu^{i}=\frac{1}{2} \epsilon^{i k s}{ }_{[k s]}^{[-1]}, \quad \stackrel{[-1]}{\mu}_{[i s]}=e_{i s j} \mu^{j} .
$$

В табл. 1 приведем псевдотензорные величины микрополярной упругости с указанием их веса и преобразования к абсолютным тензорам.

Введем микрополярный упругий потенциал $\mathscr{U}$, рассчитанный на единицу инвариантного элемента объема, с псевдотензорными аргументами (см. табл. 1):

$$
\left.\left.\mathscr{U}=\mathscr{U}\left(\epsilon_{(i j)},{ }^{[+1]}\right]_{(i j)},{ }^{[+1}\right]_{i}, \kappa_{i}\right)
$$




\section{Таблица 1}

Относительные тензоры микрополярной упругости

[Relative tensors of the micropolar elasticity]

\begin{tabular}{|c|c|c|c|}
\hline Standard terminology & Root notation & Weight & $\begin{array}{l}\text { Transformation to } \\
\text { absolute tensor }\end{array}$ \\
\hline elastic potential & $\mathscr{U}$ & 0 & - \\
\hline mass density & $\rho$ & 0 & - \\
\hline displacements vector & $u^{k}$ & 0 & - \\
\hline asymmetric strain tensor & $\epsilon_{i j}$ & 0 & - \\
\hline small strain tensor & $\varepsilon_{i j}=\epsilon_{(i j)}$ & 0 & - \\
\hline force traction vector & $t^{k}=n_{i} t^{i k}$ & 0 & - \\
\hline force stress tensor & $t^{i k}, \sigma^{i k}$ & 0 & - \\
\hline body forces & $X^{k}$ & 0 & - \\
\hline couple traction pseudovector & $m_{k}=n_{i} \mu_{\cdot k}^{i}$ & -1 & $m_{k}=e^{[-1]} m_{k}$ \\
\hline couple stress pseudotensor & $\mu_{\cdot k}^{i \cdot}$ & -1 & $\mu_{\cdot k}^{i \cdot}=e^{[-1]_{i}} \mu_{\cdot k}$ \\
\hline associated couple stress vector & $\mu^{i}$ & 0 & - \\
\hline associated couple stress pseudovector & $\tau_{k}$ & -1 & $\tau_{k}=e^{[-1]} \tau_{k}$ \\
\hline body couples & $Y_{k}$ & -1 & $Y_{k}=e^{[-1]} Y_{k}^{[-1]}$ \\
\hline coefficient of microinertia & $\Im$ & -2 & $\Im=e^{2} \stackrel{[-2]}{\Im}$ \\
\hline microrotation tensor & $\Omega_{i k}$ & 0 & - \\
\hline microrotation pseudovector & $\phi^{i}$ & +1 & $\phi^{i}=\frac{1}{e}^{[+1]}{ }^{i}$ \\
\hline wryness pseudotensor & $\kappa_{i \cdot}^{\cdot s}$ & +1 & $\kappa_{i \cdot}^{\cdot s}=\frac{1[+1]}{e} \kappa_{i \cdot s}$ \\
\hline associated wryness vector & $\kappa_{i}$ & 0 & - \\
\hline
\end{tabular}


где

$$
\epsilon_{i j}=\nabla_{i} u_{j}-\epsilon_{i j k} \stackrel{[+1]}{\varphi}, \quad \stackrel{[+1]}{\varphi}^{i}=\stackrel{[+1]}{\phi}{ }^{i}-\frac{1}{2} \epsilon^{i k l} \nabla_{k} u_{l}, \quad \kappa_{i}=\frac{1}{2} \epsilon_{i j s}{ }^{[+1]^{[}}{ }^{[j s]}
$$

Обычно аргументами упругого потенциала выступают абсолютные тензоры. Здесь существенным является использование формализма псевдотензоров, обеспечивающего чувствительность определяющих псевдоскаляров к преобразованиям инверсии пространства и зеркальным отражениям.

Упругий потенциал $\mathscr{U}$ по физическому смыслу является объективной величиной и не может меняться при повороте осей системы координат. Поэтому он (так же как и его первая вариация $\delta \mathscr{U}$ ) является абсолютным скаляром. Первая вариация упругого потенциала представляется сбалансированной по весам суммой

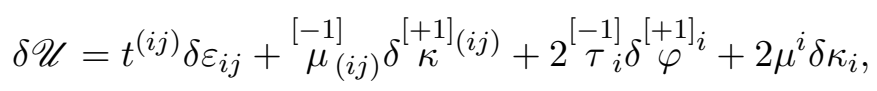

откуда могут быть получены определяющие уравнения:

$$
t^{(i j)}=\frac{\partial \mathscr{U}}{\partial \varepsilon_{i j}}, \quad \stackrel{[-1]}{\mu}_{(i j)}=\frac{\partial \mathscr{U}}{\partial^{[+1]}{ }_{\kappa}(i j)}, \quad 2^{[-1]} \tau_{i}=\frac{\partial \mathscr{U}}{\partial^{[+1]}{ }^{[+1}}, \quad 2 \mu^{i}=\frac{\partial \mathscr{U}}{\partial \kappa_{i}} .
$$

В качестве потенциала $\mathscr{U}$, который, как указывалось выше, инвариантен относительно поворотов и переносов пространства, а также относительно преобразований инверсии пространства и зеркальных отражений, в гемитропном случае следует выбрать квадратичную функцию

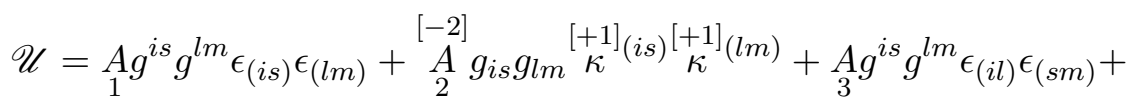

$$
\begin{aligned}
& +\stackrel{[-2]}{A} g_{i s} g_{l m} \stackrel{[+1]}{\kappa}(i l){ }_{\kappa}^{[+1]}(s m)+\stackrel{[-2]}{A} g_{i s} \stackrel{[+1]_{i}}{\varphi} i_{\varphi}^{[+1]_{s}}+\underset{6}{A} g^{i s} \kappa_{i} \kappa_{s}+ \\
& +\stackrel{[-1]}{A} g^{i s} g_{l m} \epsilon_{(i s)} \stackrel{[+1]}{\kappa}(l m)+\stackrel{[-1]}{A} \epsilon_{(i s)} \stackrel{[+1]}{\kappa}(i s)+\stackrel{[-1]}{A} \kappa_{i}^{[}{ }^{[+1} \varphi^{i},
\end{aligned}
$$

$[W]$

где символы $\underset{i}{A}(i=1, \ldots, 9)$ с соответствующими весами - определяющие псевдоскаляры гемитропной микрополярной среды (в табл. 2 представлены определяющие псевдоскаляры с указанием их веса), из них $\underset{7}{A}, \underset{8}{A}, \underset{9}{A} \underset{4}{A}$ чувствительны к преобразованиям инверсии пространства и зеркальным отражениям.

Важно отметить, что псевдоскаляры $\underset{2}{A}, \underset{4}{A}, \underset{5}{A}, \underset{7}{A}, \underset{8}{A}, \underset{9}{A}, \underset{7}{A}$, в отличие от абсолютных скаляров $\underset{1}{A}, \underset{3}{A}, \underset{6}{A}$, меняются при переходе от одной системы координат $x^{k}$ к другой системе $\bar{x}^{k}(k=1,2,3)$ в соответствии с правилом (1) 
Таблица 2

Веса микрополярных гемитропных определяющих скаляров

[The weights of the micropolar hemitropic constitutive scalars]

\begin{tabular}{|c|c|c|c|}
\hline Standard terminology & Root notation & Weight & $\begin{array}{l}\text { Transformation to } \\
\text { absolute tensor }\end{array}$ \\
\hline shear modulus of elasticity & $G$ & 0 & - \\
\hline Poisson ratio & $\nu$ & 0 & - \\
\hline $\begin{array}{l}\text { characteristic length } \\
\text { of micropolar theory }\end{array}$ & $L$ & -1 & $L=e^{[-1]} L^{[-1}$ \\
\hline first micropolar modulus & $c_{1}$ & -2 & $c_{1}=e^{2^{[-2]}} c_{1}$ \\
\hline second micropolar modulus & $c_{2}$ & +2 & $c_{2}=\frac{1}{e^{2}} \stackrel{[+2]}{c_{2}}$ \\
\hline third micropolar modulus & $c_{3}$ & 0 & - \\
\hline forth micropolar modulus & $c_{4}$ & 0 & - \\
\hline fifth micropolar modulus & $c_{5}$ & 0 & - \\
\hline sixth micropolar modulus & $c_{6}$ & 0 & - \\
\hline first constitutive pseudoscalar & $\begin{array}{c}A \\
1\end{array}$ & 0 & - \\
\hline second constitutive pseudoscalar & $\underset{2}{A}$ & -2 & $\underset{2}{A}=e^{2}{ }_{2}^{[-2]}$ \\
\hline third constitutive pseudoscalar & $\begin{array}{l}A \\
3\end{array}$ & 0 & - \\
\hline firth constitutive pseudoscalar & $\begin{array}{c}A \\
4\end{array}$ & -2 & $\underset{4}{A}=e^{2}{ }_{4}^{[-2]}$ \\
\hline fifth constitutive pseudoscalar & $\underset{5}{A}$ & -2 & $\underset{5}{A}=e^{2}{ }_{5}^{[-2]}$ \\
\hline sixth constitutive pseudoscalar & $\underset{6}{A}$ & 0 & - \\
\hline seventh constitutive pseudoscalar & $\begin{array}{c}A \\
7\end{array}$ & -1 & $\underset{7}{A}=e \underset{7}{A}$ \\
\hline eighth constitutive pseudoscalar & $\underset{8}{A}$ & -1 & $\underset{8}{A}=e \underset{8}{A}$ \\
\hline ninth constitutive pseudoscalar & $\begin{array}{l}A \\
9\end{array}$ & -1 & $\underset{9}{A}=e \underset{9}{A}$ \\
\hline
\end{tabular}


по формулам

$$
\begin{aligned}
& \underset{2}{\bar{A}}=\Delta^{-2} \underset{2}{A}, \quad \underset{4}{[-2]}=\Delta^{-2} \underset{4}{A}, \quad \underset{5}{\bar{A}}=\Delta^{-2} \underset{5}{A},
\end{aligned}
$$

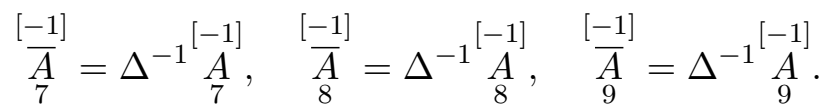

$[W]$

В декартовых системах координат определяющие псевдоскаляры $\underset{i}{A}$ остаются неизменными, может меняться только их знак. В этом случае соотношения (9) примут вид

$$
\begin{aligned}
& \underset{2}{\bar{A}}=\underset{2}{\stackrel{[-2]}{A}}, \quad \underset{4}{\bar{A}}=\underset{4}{A}, \quad \underset{5}{\bar{A}}=\underset{5}{A}, \\
& \underset{7}{\bar{A}}= \pm \underset{7}{A}, \quad \underset{[-1]}{A}= \pm \underset{8}{A} \underset{8}{A}, \quad \underset{9}{A}= \pm \underset{9}{A} .
\end{aligned}
$$

При выводе уравнений стандартной модели гемитропного микрополярного континуума в качестве определяющих постоянных принимаются постоянные абсолютные скаляры. Перейти к абсолютным скалярам в выражении (8) можно согласно правилу (3), тогда

$$
\begin{aligned}
& \underset{2}{A}=e^{2} \underset{2}{[-2]}, \quad \underset{4}{A}=e^{2} \underset{4}{A}, \quad \underset{5}{A}=e^{2}{ }_{5}^{[-2]}, \\
& \underset{7}{A}=e{\underset{7}{A}}_{7}^{A}, \quad \underset{8}{A}=e \underset{8}{A}, \quad \underset{9}{A}=e \underset{9}{A} \text {. }
\end{aligned}
$$

Воспользовавшись формулой (10), потенциал (8) можно представить в виде

$$
\begin{aligned}
& \mathscr{U}=\underset{1}{A} g^{i s} g^{l m} \epsilon_{(i s)} \epsilon_{(l m)}+e_{2}^{-2} \underset{2}{A} g_{i s} g_{l m}{ }^{[+1]} \kappa_{(i s)}^{[+1]} \kappa_{(l m)}+\underset{3}{A} g^{i s} g^{l m} \epsilon_{(i l)} \epsilon_{(s m)}+ \\
& +e^{-2} \underset{4}{A} g_{i s} g_{l m}{ }^{[+1]_{(i l)}}{ }^{[+1]} \kappa^{(s m)}+e^{-2} \underset{5}{A} g_{i s} \delta^{\left[+1 \varphi^{2}\right.} \delta^{[+1 \varphi}+\underset{6}{A} g^{i s} \kappa_{i} \kappa_{s}+ \\
& +e^{-1} \underset{7}{A} g^{i s} g_{l m} \epsilon_{(i s)}{ }^{[+1]}(\operatorname{lm})+e^{-1} \underset{8}{A} \epsilon_{(i s)} \stackrel{[+1]}{\kappa}(i s)+e^{-1} \underset{9}{A} \kappa_{i}{ }^{[+1]} \varphi_{i} .
\end{aligned}
$$

Выберем систему координат, подчинив ее условию1

$$
\sqrt{g}=1
$$

т.e.

$$
e=\operatorname{sgn} e \text {. }
$$

\footnotetext{
${ }^{1}$ Ограничение $\sqrt{g}=1$ часто используется не только в теории относительности [23], но и в механике деформируемого твердого тела [24]. На страницах 135-142 монографии [23] условие $\sqrt{g}=1$ используется при выводе уравнения тяготения в 4-пространстве-времени, что существенно упрощает уравнения теории относительности.
} 
В трехмерном пространстве таких систем существует бесконечно много, например декартовы лево- и правоориентированные системы координат. Потенциал (11) для таких систем преобразуется к виду

$$
\begin{aligned}
& \mathscr{U}=\underset{1}{A} g^{i s} g^{l m} \epsilon_{(i s)} \epsilon_{(l m)}+\underset{2}{A} g_{i s} g_{l m} \stackrel{[+1]_{(i s)}}{\kappa} \kappa_{\kappa}^{[+1]}(l m)+\underset{3}{A} g^{i s} g^{l m} \epsilon_{(i l)} \epsilon_{(s m)}+ \\
& +\underset{4}{A} g_{i s} g_{l m}{ }^{[+1]}{ }_{(i l)} \stackrel{[+1]}{\kappa}(s m)+\underset{5}{A} g_{i s}{\stackrel{[+1]_{i}}{\varphi}}^{[+1]} \varphi+\underset{6}{A} g^{i s} \kappa_{i} \kappa_{s}+
\end{aligned}
$$

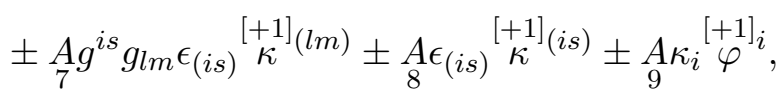

где "+" соответствует правоориентированной системе координат, а знак "_" левоориентированной; $\underset{1}{A}, \ldots, \underset{9}{A}$ являются постоянными.

Определяющие уравнения для силовых и моментных напряжений в терминах псевдотензоров в криволинейной системе координат получаются в виде

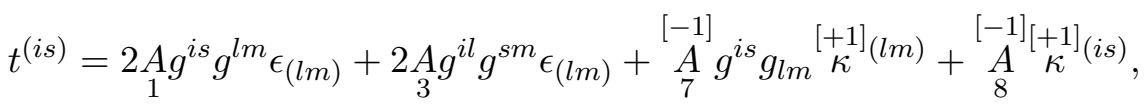

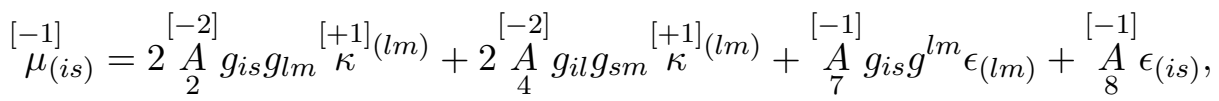

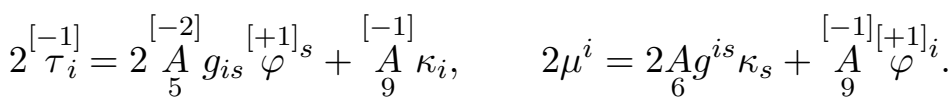

Вместо девяти определяющих псевдоскаляров $\underset{i}{A}$, появляющихся в выражении для упругого потенциала (8), удобнее ввести другие определяющие псевдоскаляры:

$$
\begin{aligned}
& \underset{1}{A}=G \nu(1-2 \nu)^{-1}, \quad \underset{2}{A}=G \stackrel{[-1][-1]}{L} L c_{3}, \quad \underset{3}{A}=G, \\
& \stackrel{[-2]}{A}=G^{[-1][-1]} L, \quad \stackrel{[-2]}{A} L=2 G^{[-2]} c_{1}, \quad \underset{6}{A}=G^{[-1][-1]} L^{[+2]} c_{2}, \\
& \stackrel{[-1]}{A}=G^{[-1]} L^{L} c_{4}, \quad \stackrel{[-1]}{A}=G^{[-1]} c_{5}, \quad \stackrel{[-1]}{A}=G^{[-1]} c^{[} c_{6},
\end{aligned}
$$

с тем чтобы в итоге пришлось иметь дело с двумя размерными и семью безразмерными параметрами:

- $G$ - модуль сдвига (имеет размерность силовых напряжений);

- $\nu$ - коэффициент Пуассона (не имеет физической размерности); $[-1]$

- $L$ - характеристическая микродлина; $[-2] \quad[+2]$

- $c_{1}, c_{2}, c_{3}, c_{4}, c_{5}, c_{6}$ - не имеющие физической размерности псевдоскаляры. 
В результате вместо (12) приходим к определяющим уравнениям гемитропной микрополярной среды:

$$
\begin{aligned}
& t^{(i s)}=2 G\left(\nu(1-2 \nu)^{-1} g^{i s} g^{l m}+g^{i l} g^{s m}\right) \epsilon_{(l m)}+G^{[-1]} L^{[-1]}\left(c_{4} g^{i s} g_{l m}{ }^{[+1]}(l m)+c_{5}^{[+1]} \kappa^{(i s)}\right) \text {, }
\end{aligned}
$$

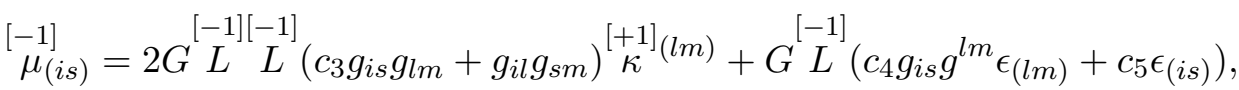

$$
\begin{aligned}
& { }^{[-1]} \tau_{i}=2 G^{[-2]} c_{1} g_{i s} \stackrel{[+1]^{\varphi}}{\varphi}+\frac{1}{2} G \stackrel{[-1]}{L} c_{6} \kappa_{i}, \quad \mu^{i}=G^{[-1][-1]} L \stackrel{[+2]}{L} c_{2} g^{i s} \kappa_{s}+\frac{1}{2} G^{[-1]} L^{[+1]_{i}} c^{[} \text {. }
\end{aligned}
$$

3. Уравнения динамики гемитропного микрополярного тела. Псевдотензорная формулировка. Уравнения динамики гемитропного микроплярного упругого континуума в криволинейных координатах, используя обозначения для дифференциальных операторов $\mathscr{L}^{i}$ и $\stackrel{[}{\mathscr{M}}_{i}$ и для определяющих постоянных

$$
c_{4}^{\prime}=c_{4}+\frac{1}{2} c_{5}+\frac{1}{4} c_{6}, \quad c_{5}^{\prime}=\frac{1}{2} c_{5}-\frac{1}{4} c_{6}, \quad c_{6}^{\prime}=-c_{6},
$$

запишем в форме

$$
\begin{aligned}
& \mathscr{L}^{i}\left(\partial ., \nabla_{k}, u^{k}, \stackrel{[+1]}{\phi}{ }^{k}\right)=G\left[\left(1+e^{2[-2]} c_{1}\right) \nabla^{s} \nabla_{s} u^{i}+\right. \\
& +\left(1-e^{2[-2]} c_{1}+2 \nu(1-2 \nu)^{-1}\right) \nabla^{i} \nabla_{k} u^{k}+ \\
& +2^{[-2]} c_{1} \epsilon^{i k l} \nabla_{k}{ }^{[+1]} \phi_{l}+{ }^{[-1]} c_{4}^{\prime} \nabla^{i} \nabla_{k}{ }^{[+1]}{ }^{k}+ \\
& \left.+{ }^{[-1]}{ }^{\prime} c_{5}^{\prime} \nabla^{k} \nabla_{k}{ }^{[+1]}{ }^{i}\right]-\rho \partial_{. .}^{2} u^{i}=0 \\
& \stackrel{[-1]}{\mathscr{M}_{i}}\left(\partial ., \nabla_{k}, u^{k}, \stackrel{[+1]}{\phi} k\right)=G^{[-1][-1]} L{ }^{L}\left[\left(1+e^{-2^{[+2]}} c_{2}\right) \nabla^{s} \nabla_{s}{ }^{[+1]} \phi_{i}+\right.
\end{aligned}
$$$$
\begin{aligned}
& +\left(1-e^{-2^{[+2]}} c_{2}+2 c_{3}\right) \nabla_{i} \nabla_{k}{ }^{[+1]} k+{ }^{[-1]} L^{-1} c_{4}^{\prime} \nabla_{i} \nabla^{k} u_{k}+ \\
& \left.+{ }^{[-1]}{ }^{-1} c_{5}^{\prime} \nabla^{k} \nabla_{k} u_{i}+{ }^{[-1]}{ }^{-1} c_{6}^{\prime} \epsilon_{i s l} \nabla^{s^{[+1]}}{ }^{l]}\right]- \\
& -2 G^{[-2]} c_{1}\left(2^{[+1]} \phi_{i}-e^{2} \epsilon_{i k l} g^{k s} \nabla_{s} u^{l}\right)-\rho \stackrel{[-2]}{\mathfrak{I}} \partial_{. .}^{2} \phi_{i}^{[+1]}=\stackrel{[-1]}{0} .
\end{aligned}
$$

На основании данных выше определений дифференциальный оператор $[-1]$

$\mathscr{L}^{i}$ имеет нулевой вес, а оператор $\mathscr{M}_{i}$ имеет вес -1 . Для правоориентиро- 
ванной декартовой системы координат уравнения (13) запишутся в виде (см. также табл. $3^{2}$ )

$$
\begin{aligned}
\mathscr{L}_{i}\left(\partial ., \partial_{k}, u_{k}, \mho_{k}\right) & =G\left[\left(1+c_{1}\right) \partial_{s} \partial_{s} u_{i}+\left(1-c_{1}+2 \nu(1-2 \nu)^{-1}\right) \partial_{i} \partial_{k} u_{k}+\right. \\
& \left.+2 c_{1} \epsilon_{i k l} \partial_{k} \mho_{l}+L c_{4}^{\prime} \partial_{i} \partial_{k} \mho_{k}+L c_{5}^{\prime} \partial_{k} \partial_{k} \mho_{i}\right]-\rho \partial_{. .}^{2} u_{i}=0, \\
\mathscr{M}_{i}\left(\partial ., \partial_{k}, u_{k}, \mho_{k}\right) & =G L^{2}\left[\left(1+c_{2}\right) \partial_{s} \partial_{s} \mho_{i}+\left(1-c_{2}+2 c_{3}\right) \partial_{i} \partial_{k} \mho_{k}+\right. \\
& \left.+L^{-1} c_{4}^{\prime} \partial_{i} \partial_{k} u_{k}+L^{-1} c_{5}^{\prime} \partial_{k} \partial_{k} u_{i}+L^{-1} c_{6}^{\prime} \epsilon_{i s l} \partial_{s} \mho_{l}\right]- \\
& -2 G c_{1}\left(2 \mho_{i}-\epsilon_{i k l} \partial_{k} u_{l}\right)-\rho \Im \partial_{. .}^{2} \mho_{i}=0 .
\end{aligned}
$$

Рассмотрим преобразование инверсии пространства $\left(x_{k} \rightarrow x_{*}\right)$. Величины в новой системе координат обозначим звездочкой снизу. В табл. 3 укажем объекты в правоориентированной декартовой системе координат, инверсной к ней системе координат и формулы связи. На основании уравнений (13), заменяя материальные постоянные в соответствии с табл. 3, получаем

$$
\begin{aligned}
& \mathscr{L}_{*}\left(\partial ., \partial_{*}^{\partial_{k}}, \underset{*}{u_{k}}, \underset{*}{\mho_{k}}\right)=G\left[\left(1+c_{1}\right) \underset{*}{\partial_{s} \partial_{*}} u_{*}+\left(1-c_{1}+2 \nu(1-2 \nu)^{-1}\right) \underset{*}{\partial_{i}} \partial_{k} u_{*}+\right. \\
& \left.+2 c_{1} \epsilon_{i k l} \partial_{*} \mho_{*} \mho_{l}-L c_{4}^{\prime} \partial_{*} \partial_{k} \mho_{*} \mho_{k}-L c_{5}^{\prime} \partial_{k} \partial_{*} \partial_{*} \mho_{i}\right]-\rho \partial_{. .}^{2} u_{*}=0, \\
& \mathscr{M}_{i}\left(\partial ., \partial_{*}, u_{*}, \mho_{*}\right)=G L^{2}\left[\left(1+c_{2}\right) \underset{*}{\partial_{s}} \partial_{*} \mho_{*} \mho_{i}+\left(1-c_{2}+2 c_{3}\right){\underset{*}{i}}_{*} \partial_{k} \mho_{*}-\right. \\
& \left.-L^{-1} c_{4}^{\prime} \partial_{i} \partial_{k} u_{*} u_{k}-L^{-1} c_{5}^{\prime} \partial_{k} \partial_{*} \partial_{*} u_{i}-L^{-1} c_{6}^{\prime} \epsilon_{i s l} \partial_{*} \mho_{*} \mho_{l}\right]- \\
& -2 G c_{1}\left(2 \mho_{*}-\epsilon_{i k l} \partial_{*} u_{*}\right)-\rho \Im \partial_{. .}^{2} \mho_{*}=0 .
\end{aligned}
$$

Производя в дифференциальных операторах замены в соответствии с третьим столбцом табл. 3, находим

$$
\begin{aligned}
& \mathscr{L}_{*}\left(\partial ., \partial_{*},{\underset{*}{*}}_{*}, \mho_{*} \mho_{k}\right)=-\mathscr{L}_{i}\left(\partial ., \partial_{k}, u_{k}, \mho_{k}\right), \\
& \mathscr{M}_{*}\left(\partial ., \partial_{*}, \underset{*}{u_{k}}, \underset{*}{\mho_{k}}\right)=\mathscr{M}_{i}\left(\partial ., \partial_{k}, u_{k}, \mho_{k}\right) .
\end{aligned}
$$

Откуда следует, что если выполняются уравнения (14) для исходной правоориентированной декартовой системы координат, то выполняются (в силу выполнимости (14)) и уравнения (15) в инверсной координатной системе 3 . Указанное обстоятельство влечет за собой, например, наличие прямых и зеркальных мод при распространении волн по гемитропному микрополярному упругому континууму. В случае распространения гармонических волн по гемитропной микрополярной среде оператор дифференцирования по времени в указанных выше уравнениях преобразуется по правилу $\partial_{. .}^{2} \rightarrow-\omega^{2}$, где $\omega-$ циклическая частота гармонической волны.

Заключение. В статье обсуждаются определяющие псевдоскаляры, связанные с теорией гемитропного микрополярного континуума.

1. Приводятся основные понятия алгебры псевдотензоров, включая обобщение теоремы Гамильтона-Кэли.

\footnotetext{
${ }^{2} \mho^{k}$ - специальный символ, вес которого мы не будем указывать, точно так же как это имеет место для $\epsilon$-символов.

${ }^{3}$ Справедливо и обратное утверждение.
} 
家

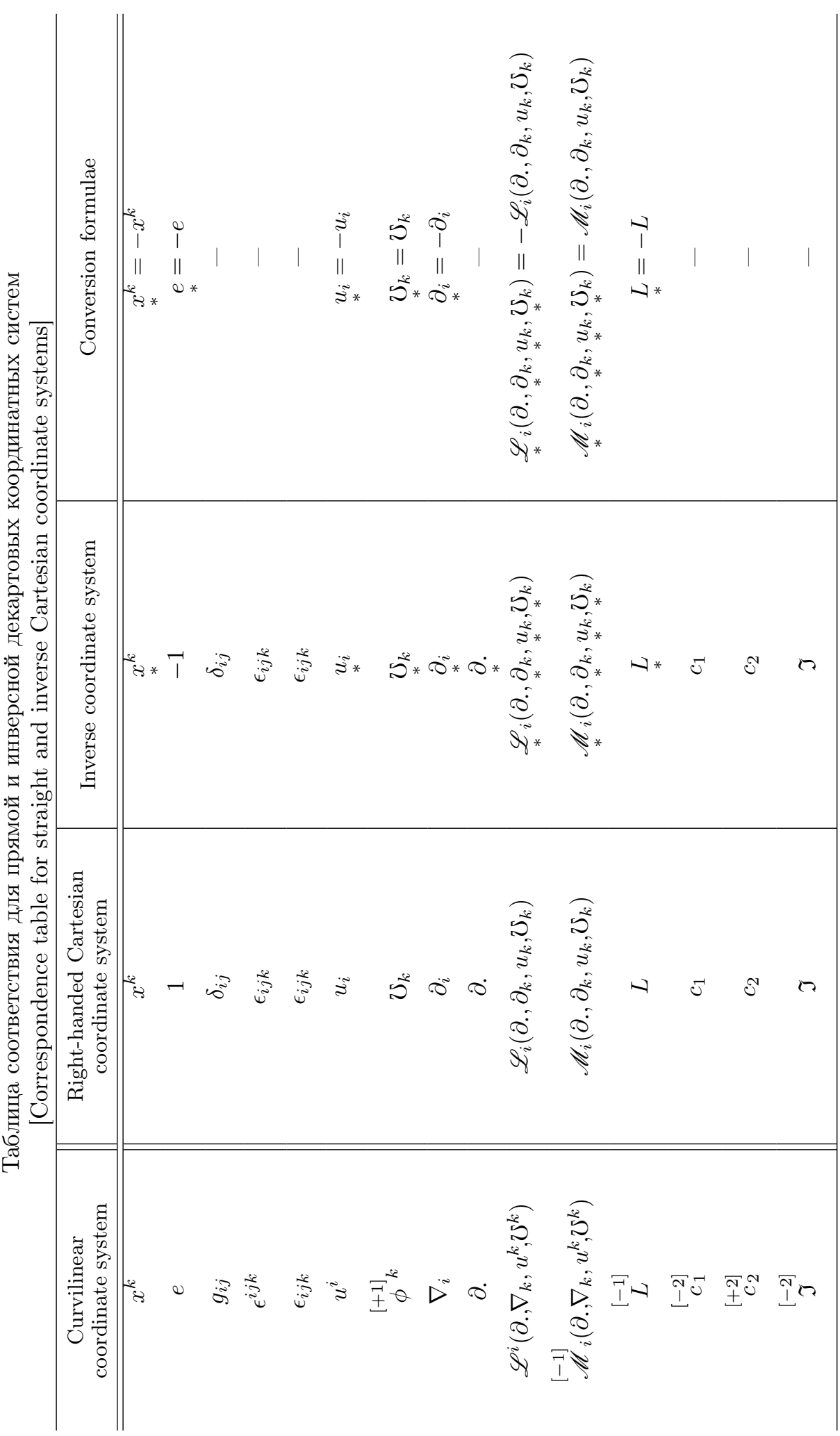


2. Определяется псевдотензорная форма гемитропного микрополярного потенциала упругой деформации, основанная на 9 определяющих псевдоскалярах (из них 3 псевдоскаляра и 6 абсолютных скаляров).

3. Вычисляются веса определяющих псевдоскаляров.

4. Продемонстрировано, что упругий потенциал инвариантен относительно поворотов и переносов пространства, а также относительно преобразований инверсии пространства и зеркальных отражений, а опреде$[-1][-1][-1]$

ляющие псевдоскаляры $A, \underset{8}{A}, \underset{9}{A}$ чувствительны к преобразованиям инверсии пространства и зеркальным отражениям.

5. Выводятся определяющие уравнения гемитропного микрополярного упругого континуума. С помощью фундаментального ориентирующего скаляра веса +1 формулируются правила преобразования определяющих псевдоскаляров.

6. Обсуждаются уравнения динамики гемитропного микрополярного континуума в терминах псевдотензоров для прямых и зеркальных мод.

Конкурирующие интересы. Конкурирующих интересов не имеем.

Авторский вклад и ответственность. Все авторы принимали участие в разработке концепции статьи; все авторы сделали эквивалентный вклад в подготовку публикации. Авторы несут полную ответственность за предоставление окончательной рукописи в печать. Окончательная версия рукописи была одобрена всеми авторами.

Финансирование. Работа выполнена в рамках государственного задания (№ госрегистрации АААА-А20-120011690132-4) и при поддержке Российского фонда фундаментальных исследований проекты № 19-51-60001, № 20-01-00666.

\section{Библиографический список}

1. Lakes R. Elastic and viscoelastic behavior of chiral materials // Int. J. Mech. Sci., 2001. vol. 43, no. 7. pp. 1579-1589. https://doi.org/10.1016/S0020-7403(00)00100-4.

2. Mackay T., Lakhtakia A. Negatively refracting chiral metamaterials: a review // SPIE Reviews, 2010. vol.1, no.1, 018003. https://doi.org/10.1117/6.0000003.

3. Tomar S., Khurana A. Wave propagation in thermo-chiral elastic medium// Appl. Math. Model., 2013. vol.37, no.22. pp. 9409-9418. https://doi.org/10.1016/j.apm.2013.04. 029.

4. Truesdell C., Toupin R. The Classical Field Theories / Principles of Classical Mechanics and Field Theory / Encyclopedia of Physics, III/1; ed. S. Flügge. Berlin, Göttingen, Heidelberg: Springer, 1960. pp. 226-902. https://doi.org/10.1007/978-3-642-45943-6_2.

5. Gurevich G. B. Foundations of the Theory of Algebraic Invariants. Groningen: P. Noordhoff, 1964. viii +429 pp.

6. McConnell A. J. Application of Tensor Analysis. New York: Dover Publ., 1957. xii +318 pp.

7. Schouten J. A. Tensor Analysis for Physicists. Oxford: Clarendon Press, 1954. xii +277 pp.

8. Sokolnikoff I. S. Tensor Analysis: Theory and Applications to Geometry and Mechanics of Continua/ Applied Mathematics Series. New York: John Wiley \& Sons Inc, 1964. xii $+361 \mathrm{pp}$.

9. Synge J. L., Schild A. Tensor Calculus/ Dover Books on Advanced Mathematics. vol. 5. New York: Courier Corporation, 1978. ix +324 pp.

10. Векуа И. Н. Основы тензорного анализа и теории ковариантов. М.: Наука, 1978. 411 с.

11. Кочин Н. Е. Векторное исчисление и начала тензорного исчисления. М.: Наука, 1951. 427 c. 
12. Радаев Ю. Н., Мурашкин Е. В. Псевдотензорная формулировка механики гемитропных микрополярных сред// Проблемы прочности и пластичности, 2020. Т. 82, № 4. C. 399-412. https://doi.org/10.32326/1814-9146-2020-82-4-399-412.

13. Murashkin E. V., Radayev Yu. N. On a micropolar theory of growing solids // Vestn. Samar. Gos. Tekhn. Univ., Ser. Fiz.-Mat. Nauki [J. Samara State Tech. Univ., Ser. Phys. Math. Sci.], 2020. vol. 24, no.3. pp. 424-444. https://doi.org/10.14498/vsgtu1792.

14. Kovalev V. A., Murashkin E. V., Radayev Yu. N. On the Neuber theory of micropolar elasticity. A pseudotensor formulation// Vestn. Samar. Gos. Tekhn. Univ., Ser. Fiz.-Mat. Nauki [J. Samara State Tech. Univ., Ser. Phys. Math. Sci.], 2020. vol. 24, no. 4. pp. 752-761. https://doi.org/10.14498/vsgtu1799.

15. Veblen O., Thomas T. Y. Extensions of relative tensors // Trans. Amer. Math. Soc., 1924. vol. 26, no. 3. pp. 373-377. https://doi.org/10.1090/S0002-9947-1924-1501284-6.

16. Veblen O. Invariants of Quadratic Differential Forms / Cambridge Tracts in Mathematics and Mathematical Physics. vol. 24. Cambridge: Univ. Press, 1927. viii +102 pp.

17. Розенфельд Б. А. Многомерные пространства. М.: Наука, 1966. 647 с.

18. Мурашкин Е. В., Радаев Ю. Н. Об одном обобщении алгебраической теории Гамильтона-Кэли // Изв. РАН. МТТ, 2021. №6 (в печати).

19. Nowacki W. Theory of Micropolar Elasticity. Course held at the Department for Mechanics of Deformable Bodies, July 1970, Udine/ International Centre for Mechanical Sciences. Courses and Lectures. vol. 25. Wien, New York: Springer, 1972. 286 pp. https://doi.org/ 10.1007/978-3-7091-2720-9.

20. Nowacki W. Theory of Asymmetric Elasticity. Oxford: Pergamon Press, 1986. viii +383 pp.

21. Besdo D. Ein Beitrag zur nichtlinearen Theorie des Cosserat-Kontinuums [A contribution to the nonlinear theory of the Cosserat-continuum]// Acta Mechanica, 1974. vol. 20, no. 1. pp. 105-131 (In German). https://doi.org/10.1007/BF01374965.

22. Радаев Ю. Н. Правило множителей в ковариантных формулировках микрополярных теорий механики континуума // Вестн. Сам. гос. техн. ун-та. Сер. Физ.-мат. науки, 2018. T. 22, № 3. C. 504-517. https://doi.org/10.14498/vsgtu1635.

23. Kopff A. The Mathematical Theory of Relativity. London: Methuen, 1923. viii +214 pp.

24. Радаев Ю. Н. Пространственная задача математической теории пластичности. Самара: Самар. ун-т, 2006. 340 с. 


\title{
MSC: 15A72, 53A45, 74D05
}

\section{On the constitutive pseudoscalars of hemitropic micropolar media in inverse coordinate frames}

\author{
(C) E. V. Murashkin, Yu. N. Radayev
}

Ishlinsky Institite for Problems in Mechanics, Russian Academy of Sciences, 101-1, pr. Vernadskogo, Moscow, 119526, Russian Federation.

\begin{abstract}
The paper is devoted to the constitutive pseudoscalars associated with the theory of hemitropic micropolar continuum. The basic concepts of pseudotensor algebra are presented. The pseudotensor form of the hemitropic micropolar elastic potential is given, based on 9 constitutive pseudoscalars ( 3 are pseudoscalars and 6 are absolute scalars). The weights of the constitutive pseudoscalars are calculated. The fundamental orienting pseudoscalar of weight +1 is used to formulate transformation rules for constitutive pseudoscalars. The governing equations of the hemitropic micropolar elastic continuum are derived. The equations of the dynamics of the hemitropic micropolar continuum are discussed in terms of pseudotensors in right- and left-handed Cartesian coordinate systems. The presence of inverse modes along with normal ones is shown for wave propagation across the hemitropic micropolar continuum.
\end{abstract}

Keywords: micropolar hemitropic continuum, microrotation, pseudoscalar, relative tensor, fundamental orienting pseudoscalar, inversion of space, polarisation.

Received: $23^{\text {rd }}$ June, 2021 / Revised: $29^{\text {th }}$ July, $2021 /$ Accepted: $25^{\text {th }}$ August, $2021 /$ First online: $27^{\text {th }}$ September, 2021

Competing interests. The authors declare no conflicts of interests.

Authors' contributions and responsibilities. Each author has participated in the article concept development; the authors contributed equally to this article. The authors are absolutely responsible for submit the final manuscript to print. Each author has approved the final version of manuscript.

\section{Research Article}

(우 (i) The content is published under the terms of the Creative Commons Attribution 4.0 International License (http://creativecommons.org/licenses/by/4.0/)

Please cite this article in press as:

Murashkin E. V., Radayev Yu. N. On the constitutive pseudoscalars of hemitropic micropolar media in inverse coordinate frames, Vestn. Samar. Gos. Tekhn. Univ., Ser. Fiz.-Mat. Nauki [J. Samara State Tech. Univ., Ser. Phys. Math. Sci.], 2021, vol. 25, no. 3, pp. 457-474. https://doi.org/10.14498/vsgtu1870 (In Russian).

\section{Authors' Details:}

Evgenii V. Murashkin (10) https://orcid.org/0000-0002-3267-4742

Cand. Phys. \& Math. Sci., PhD, MD; Senior Researcher; Lab. of Modeling in Solid Mechanics; e-mail: evmurashkin@gmail.com

Yuri N. Radayev (iD https://orcid.org/0000-0002-0866-2151

D.Sc. (Phys. \& Math. Sci.), Ph.D., M.Sc., Professor; Leading Researcher; Lab. of Modeling in Solid Mechanics; e-mail: radayev@ipmnet.ru, y.radayev@gmail.com 
Funding. This study was supported by the Ministry of Science and Higher Education of the Russian Federation (State Registration Number AAAA-A20-120011690132-4) and by the Russian Foundation for Basic Research (projects nos. 18-01-00844, 20-01-00666).

\section{References}

1. Lakes R. Elastic and viscoelastic behavior of chiral materials, Int. J. Mech. Sci., 2001, vol. 43, no. 7, pp. 1579-1589. https://doi.org/10.1016/S0020-7403(00)00100-4.

2. Mackay T., Lakhtakia A. Negatively refracting chiral metamaterials: a review, SPIE Reviews, 2010, vol.1, no. 1, 018003. https://doi.org/10.1117/6.0000003.

3. Tomar S., Khurana A. Wave propagation in thermo-chiral elastic medium, Appl. Math. Model., 2013, vol.37, no.22, pp. 9409-9418. https://doi.org/10.1016/j.apm.2013.04. 029.

4. Truesdell C., Toupin R. The Classical Field Theories, In: Principles of Classical Mechanics and Field Theory, Encyclopedia of Physics, III/1; ed. S. Flügge. Berlin, Göttingen, Heidelberg, Springer, 1960, pp. 226-902. https://doi.org/10.1007/978-3-642-45943-6_2.

5. Gurevich G. B. Foundations of the Theory of Algebraic Invariants. Groningen, P. Noordhoff, 1964, viii+429 pp.

6. McConnell A. J. Application of Tensor Analysis. New York, Dover Publ., 1957, xii +318 pp.

7. Schouten J. A. Tensor Analysis for Physicists. Oxford, Clarendon Press, 1954, xii +277 pp.

8. Sokolnikoff I. S. Tensor Analysis: Theory and Applications to Geometry and Mechanics of Continua, Applied Mathematics Series. New York, John Wiley \& Sons Inc, 1964, xii+361 pp.

9. Synge J. L., Schild A. Tensor Calculus, Dover Books on Advanced Mathematics, vol. 5. New York, Courier Corporation, 1978, ix +324 pp.

10. Vekua I. N. Osnovy tenzornogo analiza i teorii kovariantov [Foundations of Tensor Analysis and the Theory of Covariants]. Moscow, Nauka, 1978, 411 pp. (In Russian)

11. Kochin N. E. Vektornoe ischislenie i nachala tenzornogo ischisleniya [Foundations of Tensor Analysis and Covariant Theory]. Moscow, Nauka, 1951, 427 pp. (In Russian)

12. Radayev Yu. N., Murashkin E. V. Pseudotensor formulation of the mechanics of hemitropic micropolar media, Problems of Strength and Plasticity, 2020, vol. 82, no. 4, pp. 399-412 (In Russian). https://doi.org/10.32326/1814-9146-2020-82-4-399-412.

13. Murashkin E. V., Radayev Yu. N. On a micropolar theory of growing solids, Vestn. Samar. Gos. Tekhn. Univ., Ser. Fiz.-Mat. Nauki [J. Samara State Tech. Univ., Ser. Phys. Math. Sci.], 2020, vol. 24, no. 3, pp. 424-444. https://doi.org/10.14498/vsgtu1792.

14. Kovalev V. A., Murashkin E. V., Radayev Yu. N. On the Neuber theory of micropolar elasticity. A pseudotensor formulation, Vestn. Samar. Gos. Tekhn. Univ., Ser. Fiz.-Mat. Nauki [J. Samara State Tech. Univ., Ser. Phys. Math. Sci.], 2020, vol. 24, no. 4, pp. 752-761. https://doi.org/10.14498/vsgtu1799.

15. Veblen O., Thomas T. Y. Extensions of relative tensors, Trans. Amer. Math. Soc., 1924, vol. 26, no. 3, pp. 373-377. https://doi.org/10.1090/S0002-9947-1924-1501284-6.

16. Veblen O. Invariants of Quadratic Differential Forms, Cambridge Tracts in Mathematics and Mathematical Physics, vol. 24. Cambridge, Univ. Press, 1927, viii +102 pp.

17. Rozenfel'd B. A. Mnogomernye prostranstva [Multidimensional Spaces]. Moscow, Nauka, 1966, 648 pp. (In Russian)

18. Murashkin E. V., Radaev Yu. N. On a generalization of the algebraic Hamilton-Cayley theory, Mech. Solids, 2021, vol. 55, no. 6 (to appear).

19. Nowacki W. Theory of Micropolar Elasticity. Course held at the Department for Mechanics of Deformable Bodies, July 1970, Udine, International Centre for Mechanical Sciences. Courses and Lectures, vol. 25. Wien, New York, Springer, 1972, 286 pp. https://doi.org/ 10.1007/978-3-7091-2720-9.

20. Nowacki W. Theory of Asymmetric Elasticity. Oxford, Pergamon Press, 1986, viii +383 pp.

21. Besdo D. Ein Beitrag zur nichtlinearen Theorie des Cosserat-Kontinuums [A contribution to the nonlinear theory of the Cosserat-continuum], Acta Mechanica, 1974, vol. 20, no. 1, pp. 105-131 (In German). https://doi.org/10.1007/BF01374965. 
22. Radayev Yu. N. The Lagrange multipliers method in covariant formulations of micropolar continuum mechanics theories, Vestn. Samar. Gos. Tekhn. Univ., Ser. Fiz.-Mat. Nauki [J. Samara State Tech. Univ., Ser. Phys. Math. Sci.], 2018, vol. 22, no. 3, pp. 504-517 (In Russian). https://doi.org/10.14498/vsgtu1635.

23. Kopff A. The Mathematical Theory of Relativity. London, Methuen, 1923, viii +214 pp.

24. Radayev Yu. N. Prostranstvennaya zadacha matematicheskoy teorii plastichnosti [The Spatial Problem of the Mathematical Theory of Plasticity]. Samara Univ., Samara, 2006, 340 pp. (In Russian) 